\title{
Effects of Dielectric Screening Function and Image Charges on Hydrogenic Donor Binding Energy in a Surface Quantum Well
}

\author{
R. Abarna, A. Anitha and M. Arulmozhi* \\ Department of Physics, Jayaraj Annapackiam College for Women (Autonomous), \\ Periyakulam 625601, Theni District, Tamil Nadu, India \\ ${ }^{*}$ Corresponding author: arulpkm@yahoo.co.in
}

Published online: 15 April 2017

To cite this article: Abarna, R., Anitha, A. \& Arulmozhi, M. (2017). Effects of dielectric screening function and image charges on hydrogenic donor binding energy in a surface quantum well. J. Phys. Sci., 28(1), 73-85, https://doi.org/10.21315/jps2017.28.1.6

To link to this article: https://doi.org/10.21315/jps2017.28.1.6

\begin{abstract}
We have presented a theoretical study on ground state binding energy of the hydrogenic donor in the Surface Quantum Well composed of vacuum $/ G a A s / G a_{1-x} A l_{x} A s$. The effects of image charges and dielectric screening function on the hydrogenic donor binding energy are analysed. The results indicate that both of them have inverse effects. These results are compared with the results of other potential profiles previously reported.
\end{abstract}

Keywords: Surface quantum well, hydrogenic donor, binding energy, dielectric screening function, image charge

\section{INTRODUCTION}

Low dimensional semiconductor systems, especially the nature of the impurity states in quantum wells, are extensively studied because of purely scientific interest and their potential applications in the manufacturing of the opto-electronic devices. ${ }^{1}$ Binding energies of a hydrogenic donor in different quantum wells with $\mathrm{GaAs} / \mathrm{Ga}_{1-\mathrm{x}} \mathrm{Al}_{\mathrm{x}} \mathrm{As}$ have been investigated experimentally and theoretically by many authors. ${ }^{2-7}$ Several authors have reported the effect of perturbations on hydrogenic donor binding energy in different quantum wells. ${ }^{8,9}$

Surface Quantum Well (SQW) composed of vacuum/GaAs/GaAlAs are of considerable interest due to: (1) the presence of localised states even above the single quantum barrier in the GaAlAs layer; ${ }^{10}$ and (2) the image charges that arise 
from the large dielectric mismatch at the single vacuum/GaAs interface. Present authors have reported the binding energy of the excitons in such a SQW. ${ }^{11}$

The effect of dielectric screening function, which is the spatial dependent screening of an impurity ion caused by the valence electrons and the effect of dielectric mismatch at the interfaces play a very important role in the study of hydrogenic donor binding energy in low dimensional semiconductor systems.

Shallow impurity states in a freestanding semiconductor nanowire and in a semiconductor nanowire surrounded by a metallic gate have been studied by $\mathrm{Li}$ et al. ${ }^{12}$ The effects of dielectric mismatch on the binding energies of impurity in GaAs- $\mathrm{Ga}_{1-\mathrm{x}} \mathrm{Al}_{\mathrm{x}} \mathrm{As}$ quantum wells have been studied by Deng et al. ${ }^{13}$ Pereira et al. ${ }^{14}$ have investigated the electron-impurity binding energy in $\mathrm{GaN} / \mathrm{HfO}_{2}$ quantum wells considering simultaneously all energy contributions caused by the dielectric mismatch.

Niculescu has made a theoretical study of the electronic states in a spherical quantum dot with and without a hydrogenic impurity, taking into account the dielectric mismatch effect, by considering the joint action of the quantum confinement and polarisation charges. ${ }^{15}$ Exciton states in the cubic quantum dots with finite potential barrier in the presence of dielectric mismatch have been studied by Boichuk et al. ${ }^{16}$ The role of effective mass and dielectric mismatch on chemical potentials and addition energies of many-electron multi-shell quantum dots have been studied by Royo et al. ${ }^{17}$

Sukumar and Navaneethakrishnan studied the effect of dielectric function and pressure on the binding energies of excitons in GaAs and $\mathrm{GaAs} / \mathrm{Ga}_{1-\mathrm{x}} \mathrm{Al}_{\mathrm{x}} \mathrm{As}$ superlattices, whereas Deng et al. calculated the binding energies of shallow donors and acceptors in a spherical GaAs/ $\mathrm{Ga}_{1-\mathrm{x}} \mathrm{Al}_{\mathrm{x}} \mathrm{As}$ quantum dot including the spatial variation of dielectric screening. ${ }^{18,19}$ In addition, Kilicarslan et al. have shown that for $\mathrm{Si}$, there is an obvious increase in the binding energy for spatially dependent screening compared with that for constant screening in the range of considered well widths, whereas the spatially dependent screening effect is small for Ge and GaAs materials. ${ }^{20}$ The effect of spatial dielectric screening on the diamagnetic susceptibility of a donor in low dimensional semiconducting systems like quantum well, quantum well wire and quantum dot in the infinite barrier model has been investigated by Nithiananthi et al. ${ }^{21}$ The effect of the dielectric screening on donor binding energies in a quantum wire has been estimated by Latha et al. with five different screening functions. ${ }^{22}$ 
Keldysh has shown that in thin films, if the dielectric constant of the film is much larger than that of the substrate, as film thickness decreases, the Coulomb interaction increases strongly. ${ }^{23}$ Shik, on the other hand, observed a decrease of conductivity with the film thickness, when screening in thin films is taken into account. ${ }^{24}$ Chaplik has investigated the scattering of electrons by ions in an inversion layer. ${ }^{25}$

Deng and Gu have calculated the effects of the image potential on hydrogenic impurity binding energies in GaAs/AlAs quantum well wires with rectangular cross section. ${ }^{26}$ The role of dielectric constant mismatch on shallow donor impurities in GaAs- $\mathrm{Ga}_{1-\mathrm{x}} \mathrm{Al}_{\mathrm{x}} \mathrm{As}$ quantum well structures have been investigated by Fraizzoli et al. ${ }^{27}$ The effect of image charges due to dielectric mismatch on the properties of donor in semiconductor nanostructures bounded by air have been studied by Corfdir et al..$^{28}$

Wendler et al. have reported the exciton binding energy in semiconductor quantum well with the effect of the image potential. ${ }^{29}$ Thoai et al. studied the influence of image charges and discontinuous masses in quantum well on exciton binding energy. ${ }^{30}$ The effect of image charges on the ground state binding energy of hydrogenic impurity in cylindrical quantum well wire has been calculated as a function radius of the wire by Thoai. ${ }^{31}$ Elabsy has investigated the effect of image forces on the binding energy of impurity atoms placed at the centre of a GaAs$\mathrm{Ga}_{1-\mathrm{x}} \mathrm{Al}_{\mathrm{x}} \mathrm{As}$ quantum well. ${ }^{32}$ Diarra et al. have shown that ionisation energy of the impurities in semiconductor nanowires is strongly enhanced with respect to the bulk, when the wires are embedded in a material with low dielectric constant. ${ }^{33}$ Aharonyan has studied the screened Coulomb states in a finite confining potential semiconductor quantum well. ${ }^{34}$

In the present work, we have reported the effect of dielectric screening on the binding energy of a hydrogenic donor in a SQW composed of vacuum/GaAs/ GaAlAs as a function of well width and Al composition. The role of image charges which arise due to the large difference in the dielectric constants on either side of the interface between vacuum and GaAs, in such a SQW are considered.

\section{THEORY AND FORMULATION}

The Hamiltonian of a hydrogenic donor in a quantum well is given in the effective mass approximation as: ${ }^{5}$

$$
H=-\frac{1}{\rho} \frac{\partial}{\partial \rho} \rho \frac{\partial}{\partial \rho}-\frac{\partial^{2}}{\partial z^{2}}+V(z)-\frac{2}{r}
$$


We have used effective Rydbergs as the unit of energy $R^{*}=m^{*} e^{4} / 2 \in{ }_{0}^{2} \hbar^{2}$ and effective Bohr radius $a^{*}=\in{ }_{0} \hbar^{2} / m^{*} e^{2}$ as the unit of length. $\in_{0}$ is the static dielectric constant and $\mathrm{m}^{*}$ is the effective mass of electron. For GaAs, we have used $\in_{0}=13.2$ and $m^{*}=0.0665 m_{0}^{5}$, where $m_{0}$ is the free-electron mass.

The potential profile for SQW is given by

$$
V(z)=\left\{\begin{array}{lr}
\infty & z<0 \\
0 & 0<z<L \\
V_{o} & z>L
\end{array}\right.
$$

$L$ is the well width and $V_{o}$ is the barrier height calculated as $0.65 \Delta \mathrm{E}_{\mathrm{g}} .{ }^{5}$ The band gap difference $\Delta \mathrm{E}_{\mathrm{g}}$ between $\mathrm{GaAs}$ and $\mathrm{Ga}_{1-\mathrm{x}} \mathrm{Al}_{\mathrm{x}} \mathrm{As}$ is related to the $\mathrm{Al}$ concentration $\mathrm{x}$ by: ${ }^{5}$

$\Delta \mathrm{E}_{\mathrm{g}}=1.155 \mathrm{x}+0.37 \mathrm{x}^{2} \mathrm{eV}$

The trial wave function ${ }^{11}$ for ground state of the hydrogenic donor impurity state can be written as:

$\psi(r)=\left\{\begin{array}{lr}0 & z<0 \\ A \sin z e^{-a r} & 0<z<L \\ B e^{-\beta z} e^{-a r} & z>L\end{array}\right.$

where $A$ is normalisation constant and $B$ is related to $A$ as $B=A e^{\beta L} \sin L$. $\alpha$ and $\beta$ are given by:

$$
\alpha=\sqrt{\frac{2 m^{*} E_{e}}{\hbar^{2}}} \text { and } \beta=\sqrt{\frac{2 m^{*}\left(V_{o}-E_{e}\right)}{\hbar^{2}}}
$$

The transcendental equation to be solved for the electron energy levels $\left(E_{e}\right)$ is obtained by matching the wavefunction and its first derivative at $z=L$ as:

$$
\pm\left(\frac{E_{e}}{V_{0}}\right)^{1 / 2}=\sin \left(\sqrt{E_{e} L}\right)
$$

The expectation value of the Hamiltonian is calculated using the expression:

$$
\langle H\rangle=\frac{\langle\psi|H| \psi\rangle}{\langle\psi \mid \psi\rangle}
$$


Binding energy of the hydrogenic donor is determined as:

$$
E_{b}=E_{e}-\langle H\rangle_{\min }
$$

where $\langle H\rangle_{\min }$ is the minimised value of $\langle H\rangle$ with respect to the variational parameter "a."

\subsection{The Effect of Image Charges}

The image charges arise due to the different dielectric constants at the interface between vacuum and GaAs. The image charge of an electron or impurity ion is expressed as: ${ }^{35}$

$q_{i}^{\prime}=\frac{\in_{0}-\in_{1}}{\in_{0}+\in_{1}} q_{i}=K q_{i}$

where the dielectric constant in the GaAs well is $\in_{\circ}$ and that in vacuum is $\in_{1}=1$. $q_{i o n}=+e, q^{e}=-e, q_{i o n}^{\prime}=K e$ and $q_{e}^{\prime}=-K e$.

Unlike the symmetrical wells, the image charges are considered only at the single vacuum/GaAs interface and not at $\mathrm{GaAs} / \mathrm{Al}_{\mathrm{x}} \mathrm{Ga}_{1-\mathrm{x}} \mathrm{As}$ interface in the SQW. As a result, only one single image each for the electron and the impurity ion is formed at the interface. The electron sees not only the impurity ion but also its own image charge and that of the impurity ion; similar is the case of the impurity ion. The additional potential in the Hamiltonian, when the image charges are considered, is given by:

$V_{a}=\frac{q_{e} q_{i o n}^{\prime}}{\in_{0} r}+\frac{q_{i o n} q_{e}^{\prime}}{\in_{0} r}+\frac{q_{e} q_{e}^{\prime}}{\in_{0} 2_{z}}+\frac{q_{i o n} q_{i o n}^{\prime}}{2 \in{ }_{0} \rho}$

The 4th term in Equation 10 corresponds to the interaction between the impurity ion and its image which is neglected by Elabsy due to its small contribution and for simplifying the calculations, but included by Deng et al. and Deng and Gu. ${ }^{13,26,32}$ Taking the unit of energy as the effective Rydberg and the unit of length as the effective Bohr radius, the image potential now becomes:

$$
V_{a}=K\left[\frac{-4}{\sqrt{\rho^{2}+z^{2}}}+\frac{1}{z}+\frac{1}{\rho}\right]
$$

The new Hamiltonian for the hydrogenic donor is now given by:

$$
H_{\text {image }}=H+V_{a}
$$


The binding energy of the hydrogenic donor is determined by evaluating $\left\langle H_{\text {image }}\right\rangle$ and minimising it with respect to the variational parameter "a" using the trial wave function given in Equation 4. The binding energy of the hydrogenic donor is now given by:

$$
E_{b}=E_{e}-\left\langle H_{\text {image }}\right\rangle_{\min }
$$

\subsection{The Effect of Dielectric Screening Function (DSF)}

The spatial variation of dielectric constant in the quantum well i.e., the dielectric screening function is given by: ${ }^{18}$

$$
\frac{1}{\in(r)}=\frac{1}{\epsilon_{\mathrm{o}}}+\left(1-\frac{1}{\epsilon_{\mathrm{o}}}\right) e-\frac{r}{c}
$$

where $\mathrm{c}$ is the lattice constant and $r=\sqrt{\rho^{2}+z^{2}}$. For GaAs, $\mathrm{c}=0.565 \mathrm{~nm}$. When the effect of dielectric screening function is included, the Hamiltonian given in Equation 1 is modified as:

$$
H_{D S F}=-\frac{1}{\rho} \frac{\partial}{\partial \rho} \rho \frac{\partial}{\partial \rho}-\frac{\partial^{2}}{\partial z^{2}}+V(z)-\frac{2}{r}\left(\frac{\epsilon_{o}}{\in(r)}\right)
$$

Binding energy of the hydrogenic donor is determined again with this Hamiltonian using the expressions given in Section 2 (without image charges) and Section 2.1 (with image charges) as:

$$
E_{b}=E_{e}-\left\langle H_{D S F}\right\rangle_{\min }
$$

\section{RESULTS AND DISCUSSION}

The effective Bohr radius (the length unit) and the effective Rydbergs (the energy unit) are calculated as $a^{*}=10.50397 \mathrm{~nm}$ and $R^{*}=5.19275 \mathrm{meV}$ respectively. Figure 1 displays the binding energy of the hydrogenic donor as function of wellwidth $\mathrm{L}$ for the concentration of $\mathrm{Al}, \mathrm{x}=0.3$. We have performed the calculations under four cases, with and without the effect of DSF and the image charges. 


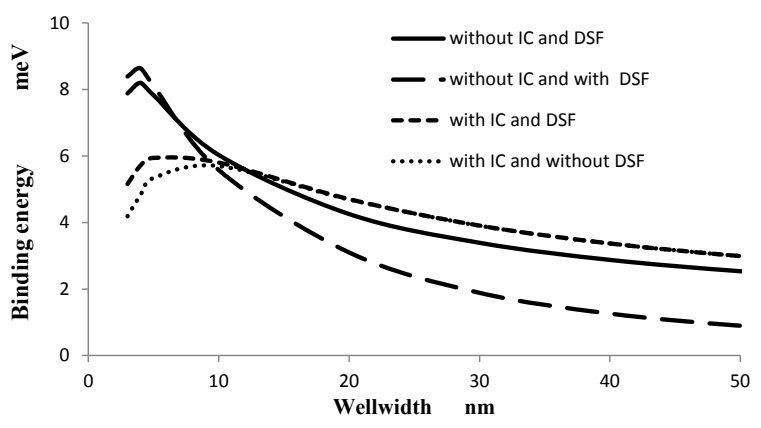

Figure1: Binding energy of hydrogenic donor vs. wellwidth (IC denotes image charges).

It is noted that in all the cases, the hydrogenic donor binding energy increases with decrease in wellwidth up to certain value $(\mathrm{L}=4 \mathrm{~nm}$ without image charges and $\mathrm{L}=5 \mathrm{~nm}$ with image charges, irrespective of DSF) due to the compression of donor wavefunction in the quantum well, and starts to decrease with further decrease in wellwidth due to the spread out of the donor wavefunction into the surrounding $\mathrm{Ga}_{1-\mathrm{x}} \mathrm{Al}_{\mathrm{x}} \mathrm{As}$ layer. Therefore, as wellwidth is reduced, a turnover is observed in the binding energy of the donor. Finite quantum wells of all shapes exhibit this behaviour. ${ }^{4-6}$ From Figure 1, it is also apparent that the image charges and not the DSF play a vital role in changing the turnover wellwidth. When the effect of image charges is included, the wavefunction spreads out earlier into the barrier and hence the turnover wellwidth increases. This behaviour is similar to that of excitons in the same SQW. ${ }^{11}$

Crossover in the values of binding energy occurs at the wellwidth $L=7 \mathrm{~nm}$ without image charges, $\mathrm{L}=10 \mathrm{~nm}$ with image charges, $\mathrm{L}=9 \mathrm{~nm}$ with DSF and $\mathrm{L}=12 \mathrm{~nm}$ without DSF. Crossover wellwidths denote the wellwidths at which there is a switchover in the domination in the effects of image charges and DSF. For example, without image charges, as wellwidth decreases, hydrogenic donor binding energy without DSF is more than that with DSF upto $\mathrm{L}=7 \mathrm{~nm}$. For $\mathrm{L}<7$ $\mathrm{nm}$, the condition changes vice versa. In contrast, the results reported earlier show that the DSF increases the confinement and hence increases the binding energy for all wellwidths. ${ }^{18,19,22}$ The reasons for this difference may be due to the asymmetric nature of the well, and the inclusion of the effect of image charges.

The peak value of donor binding energy is its limiting value before the spilling over of donor wavefunction into the single $\mathrm{Ga}_{1-\mathrm{x}} \mathrm{Al}_{\mathrm{x}} \mathrm{As}$ barrier leading to the delocalised states in it. For symmetric infinite quantum wells, as wellwidth increases donor binding energy continuously increases to $2 \mathrm{D}$ limit as $\mathrm{L} \rightarrow 0$, since $\mathrm{Ga}_{1-\mathrm{x}} \mathrm{Al} \mathrm{l}_{\mathrm{x}} \mathrm{As}$ barriers are not available for the donor wavefunction to spill over. For symmetric 
finite quantum wells, $\mathrm{Ga}_{1-\mathrm{x}} \mathrm{Al}_{\mathrm{x}} \mathrm{As}$ barrier exists on both sides of GaAs layer giving rise to two $\mathrm{GaAs} / \mathrm{Ga}_{1-\mathrm{x}} \mathrm{Al}_{\mathrm{x}} \mathrm{As}$ interfaces and as wellwidth decreases, these wells show a behaviour explained earlier in the second paragraph of this section with donor binding energy approaching $1 \mathrm{R}^{*}$ of bulk $\mathrm{Ga}_{1-\mathrm{x}} \mathrm{Al}_{\mathrm{x}} \mathrm{As}$ as $\mathrm{L} \rightarrow 0$. But in the present case of $\mathrm{SQW}$, only a single $\mathrm{GaAs} / \mathrm{Ga}_{1-x} \mathrm{Al}_{\mathrm{x}} \mathrm{As}$ interface is present. Hence the peak value is smaller than the $2 \mathrm{D}$ limit ( $4 \mathrm{R}^{*}$ of bulk GaAs) but larger than $1 \mathrm{R}^{*}$ of bulk $\mathrm{Ga}_{1-\mathrm{x}} \mathrm{Al}_{\mathrm{x}} \mathrm{As}$. These values are comparable with those of excitons in the same SQW. ${ }^{11}$

It is also noted that for $\mathrm{L}<12 \mathrm{~nm}$ without DSF and for $\mathrm{L}<9 \mathrm{~nm}$ with DSF, the effect of image charges decreases the donor binding energy. The presence of image charges is more pronounced in quantum wells with smaller wellwidths than those with larger one. In symmetric wells, when image charges are included, donor experiences repulsion due to the image charges at both interfaces which increases the confinement and hence the binding energy. Moreover, the compression of donor wavefunction in the GaAs well further increases the binding energy. Hence, the binding energy with image charges is greater than that without image charges for all wellwidths. ${ }^{23-25,33,34}$

However, in the present case, when the image charges are included, as wellwidth decreases beyond the crossover wellwidths, the competing effects of repulsion experienced at the single vacuum/GaAs interface and the compression of donor wavefunction in the GaAs well results in the decrease of donor binding energy, than when the image charges not included. This decrease in binding energy for these wellwidths increases the doping efficiency of the impurities at room temperature, reducing the need for heavy doping to obtain good electrical properties. ${ }^{33}$

Figure 2 shows the variation of ground state binding energy of hydrogenic donor as a function of wellwidth for various $\mathrm{Al}$ concentrations including the effect of DSF. It is observed that the binding energy is less sensitive to the Al concentration upto the wellwidth $\mathrm{L}=10 \mathrm{~nm}$. For $\mathrm{L}<10 \mathrm{~nm}$, it shows significant variation in binding energy. It is also noted that when the concentration of $\mathrm{Al}$ increases, the peak value of the binding energy increases and shifts towards the lower wellwidth. 


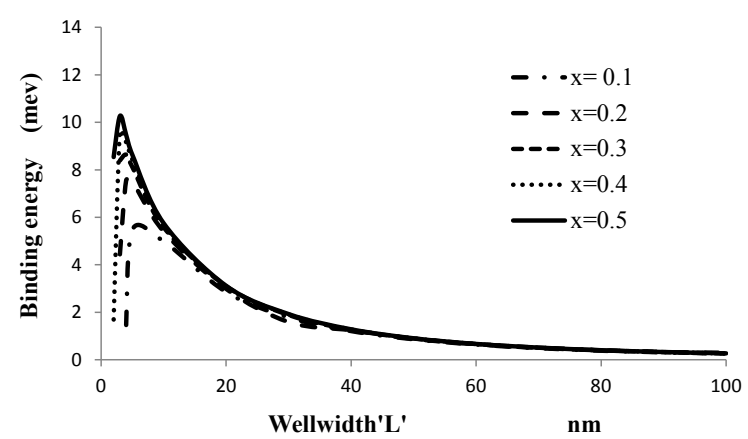

Figure 2: Binding energy as a function of wellwidth for various $\mathrm{Al}$ concentrations including the effect of dielectric screening function.

Figure 3 displays the variation of binding energy of a hydrogenic donor as a function of Al concentration for $\mathrm{L}=20 \mathrm{~nm}$. The results are similar to those reported by Deng et al. ${ }^{13}$

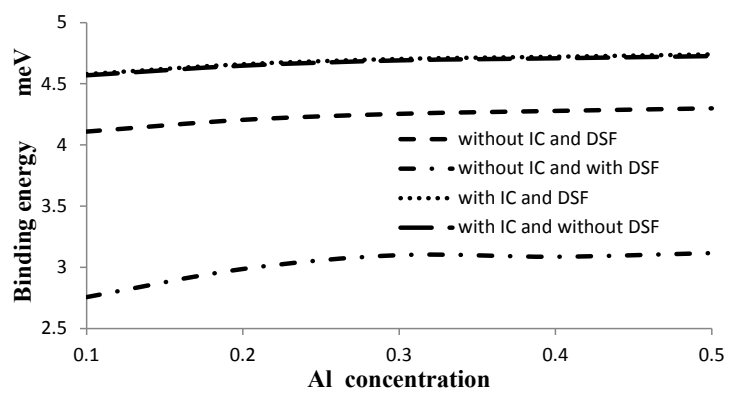

Figure 3: Binding energy as a function of $\mathrm{Al}$ concentration with and without dielectric screening function for $\mathrm{L}=20 \mathrm{~nm}$.

The following observations are noted in the present case:

- Effect of DSF is to decrease the binding energy by nearly $1.3 \mathrm{meV}$ without image charges and $0.2 \mathrm{meV}$ with image charges

- Image charges increase the binding energy with DSF by nearly $1.5 \mathrm{meV}$ and $0.4 \mathrm{meV}$ without DSF

These observations confirm that the presence of image charges increases the confinement of the hydrogenic donor and hence increases the binding energy. However, the DSF decreases the confinement and hence decreases the binding 
energy. Since random distribution of donor atoms leads to the distribution of binding energy and hence the emission energy, ${ }^{28}$ inclusion of image charges and DSF will be significant in the study of emission properties of semiconductor quantum wells.

\section{CONCLUSION}

We have calculated the ground state binding energy of hydrogenic donor in a SQW formed by vacuum/GaAs/ $/ \mathrm{Ga}_{1-\mathrm{x}} \mathrm{Al}_{\mathrm{x}} \mathrm{As}$ as a function of wellwidth and $\mathrm{Al}$ concentration. The important results obtained are that: (1) the binding energy increases when the wellwidth decreases up to a particular wellwidth and decreases for further decrease in wellwidth after reaching a peak value; (2) the peak value of the hydrogenic donor binding energy increases when the effect of DSF is considered; (3) peak value of binding energy shifts to lower wellwidth as Al concentration increases; and (4) crossover in the values of binding energy occurs at the wellwidth $\mathrm{L}=7 \mathrm{~nm}$ without image charges, $\mathrm{L}=10 \mathrm{~nm}$ with image charges, $\mathrm{L}=9 \mathrm{~nm}$ with $\mathrm{DSF}$ and $\mathrm{L}=12 \mathrm{~nm}$ without DSF.

\section{ACKNOWLEDGEMENTS}

The authors thank the University Grants Commission (UGC), New Delhi, India for the financial support through Major Research Project (No.F.42-836/2013(SR) dated 22 March 2013) and the authorities of Jayaraj Annapackiam College for Women (Autonomous), Tamil Nadu, India for the encouragements.

\section{REFERENCES}

1. Fang, W. Y. et al. (2005). On the binding energy of excitons in polar quantum well structures in a weak electric field. Chin. Phys., 14(11), 2314-2319, https://doi.org/10.1088/1009-1963/14/11/028.

2. Jarosik, N. C. et al. (1985). Binding of shallow donor impurities in quantumwell structures. Phys. Rev. Lett., 54(12), 1283-1286, https://doi.org/10.1103/ PhysRevLett.54.1283.

3. Bastard, G. (1981). Hydrogenic impurity states in a quantum well: A simple model. Phys. Rev. B, 24(8), 4714-4722, https://doi.org/10.1103/ PhysRevB.24.4714. 
4. Greene, R. L. \& Bajaj, K. K. (1985). Shallow impurity centers in semiconductor quantum well structures. Solid State Commun., 53(12), 1103-1108, https://doi.org/10.1016/0038-1098(85)90887-7.

5. Arulmozhi, M. \& Balasubramanian, S. (1994). Binding energy of a hydrogenic donor and of a wannier exciton in the $|\mathrm{z}|^{2 / 3}$ quantum well. Phys. Rev. B, 51(4), 2592-2595, https://doi.org/10.1103/PhysRevB.51.2592.

6. El-Meshad, N., Hassanien, H. M. \& Hassan, H. H. (2001). Donor binding energy in a parabolic quantum well. Fiz. A, 10, 13-20.

7. Sadeghi, E. \& Khorded, R. (2006). Hydrogenic impurity in ridge quantum wire. Braz. J. Phys., 36(4a), 1213-1216, https://doi.org/10.1590/S010397332006000700017.

8. Morales, A. L. et al. (2002). Simultaneous effects of hydrostatic stress and an electric field on donors in a GaAs-(Ga,Al)As quantum well. J. Phys. Condens. Matt., 14(5), 987-995, https://doi.org/10.1088/0953-8984/14/5/304.

9. Elabsy, A. M. (1992). Temperature dependence of shallow donor states in GaAs- $\mathrm{Al}_{\mathrm{x}} \mathrm{Ga}_{1-\mathrm{x}} \mathrm{As}$ compositional superlattice. Phys. Script., 46(5), 473-475, https://doi.org/10.1088/0031-8949/46/5/015.

10. Parks, C. et al. (1994). Observation of electronic states confined in surface quantum wells and above quantum barriers with modulated reflectivity. Solid. State Commun., 92(7), 563-567, https://doi.org/10.1016/00381098(94)00625-3.

11. Arulmozhi, M. \& Anitha, A. (2014). Excitons in a surface quantum well. Superlatt. Microstruct., 75, 222-232, https://doi.org/10.1016/j. spmi.2014.07.027.

12. Li, B. et al. (2008). Dielectric mismatch effect on coupled shallow impurity states in a semiconductor nanowire. Phys. Rev. B, 79, 085306, https://doi. org/10.1103/PhysRevB.79.085306.

13. Deng, Z. Y., Lai, T. T. \& Guo, J. K. (1994). Effects of dielectric mismatch on the impurity binding energies in GaAs-GaAlAs quantum wells. Phys. Rev. B, 50(8), 5732-5735, https://doi.org/10.1103/PhysRevB.50.5732.

14. Pereira, T. A. S. et al. (2015). Dielectric mismatch and shallow donor impurities in $\mathrm{GaN} / \mathrm{HfO}_{2}$ quantum wells. Phys. E, 66, 81-86, https://doi. org/10.1016/j.physe.2014.10.005.

15. Niculescu, E. C. (2011). Dielectric mismatch effect on the photoionization cross section and intersublevel transitions in GaAs nanodots. Opt. Comm., 284, 3298-3303, https://doi.org/10.1016/j.optcom.2011.02.071.

16. Boichuk, V. I. et al. (2011). Dielectric mismatch at finite barrier cubic quantum dots. J. Phys. Conf. Series, 289, 012004(1)-012004(14), https:// doi.org/10.1088/1742-6596/289/1/012004. 
17. Royo, M., Planelles, J. \& Pi, M. (2007). Effective mass and dielectric constant mismatch effects in spherical multishell quantum dots. Phys. Rev. $B$, 75, Article ID 033302, https://doi.org/10.1103/physrevb.75.033302.

18. Sukumar, B. \& Navaneethakrishnan, K. (1990). Effect of the dielectric function and pressure on the binding energies of excitons in GaAs and GaAs/GaAlAs superlattices. Solid State Commun., 76(4), 561-564, https:// doi.org/10.1016/0038-1098(90)90670-7.

19. Deng, Z. Y., Guo, J. K. \& Lai, T. T. (1994). Impurity states in a spherical GaAsGaAlAs quantum dot: Effects of the spatial variation of dielectric screening. Phys. Rev. B, 50(8), 5736-5739, https://doi.org/10.1103/PhysRevB.50.5736.

20. Kilicarslan, E. et al. (2010). The effects of the magnetic field and dielectric screening on the diamagnetic susceptibility of a donor in a quantum well with anisotropic effective mass. Phys. E, 42(5), 1531-1535, https://doi. org/10.1016/j.physe.2009.12.031.

21. Nithiananthi, P., Vickraman, P. \& Jayakumar, K. (2009). Effect of dielectric screening on the diamagnetic susceptibility of a donor in low dimensional semiconducting systems. Int. J. Modern Phys. B, 23(8), 2069-2075, https:// doi.org/10.1142/S0217979209052108.

22. Latha, M., Rajashabala, S. \& Navaneethakrishnan, K. (2006). Effect of dielectric screening on the binding energies and diamagnetic susceptibility of a donor in a quantum well wire. Phys. Stat. Sol. B, 243(6), 1219-1228, https://doi.org/10.1002/pssb.200541395.

23. Keldysh, L. V. (1979). Coulomb interaction in thin semiconductor and semimetal films. JETP Lett., 29(11), 658-661.

24. Shik, A. Y. (1969). Scattering of carriers by charge centers under conditions of quantum size effect. Phys. Stat. Sol., 34, 661-664, https://doi.org/10.1002/ pssb.19690340227.

25. Chaplik, A. V. (1971). Energy spectrum and electron scattering process in inversion layers. Soviet Phys., 33(5), 997-999.

26. Deng, Z. Y. \& Gu, S. (1993). Effects of image potential on hydrogenic impurity states in quantum well wires. Phys. Lett. A, 174(4), 320-324, https://doi.org/10.1016/0375-9601(93)90147-R.

27. Fraizzoli, S., Bassani. F. \& Buczko, R. (1990). Shallow donor impurities in GaAs- $\mathrm{Ga}_{1-\mathrm{x}} \mathrm{Al}_{\mathrm{x}} \mathrm{As}$ quantum well structures: Role of the dielectric constant mismatch, Phys. Rev. B, 42(8), 5096-5103, https://doi.org/10.1103/ PhysRevB.41.5096.

28. Corfdir, P. \& Lefebvre, P. (2012). Role of the dielectric mismatch on the properties of donors in semiconductor nanostructures bounded by air. J. Appl. Phys., 112(10), 106104(1)-106104(3), https://doi.org/10.1063/1.4765031. 
29. Wendler, L. \& Hartwig, B. (1991). Effects of the image potential on the binding energy of excitons in semiconductor quantum wells. $J$. Phys. Condens. Matt., 3, 9907-9914, https://doi.org/10.1088/0953-8984/3/49/007.

30. Thoai, D. B. T. et al. (1990). Image charges in semiconductor quantum wells: Effects on exciton binding energy. Phys. Rev. B, 42(9), 5909-5909.

31. Thoai, D. B. T. (1992). Hydrogenic impurity in quantum well wires/Effects of the image charges. Solid. State Commun., 81(11), 945-947, https://doi. org/10.1016/0038-1098(92)90873-8.

32. Elabsy, A. M. (1992). Effect of image forces on the biding energies of impurity atoms in $\mathrm{Ga}_{1-x} \mathrm{Al}_{\mathrm{x}} \mathrm{As} / \mathrm{GaAs} / \mathrm{Ga}_{1-x} \mathrm{Al}_{\mathrm{x}} \mathrm{As}$ quantum wells. Phys. Rev. B, 46(4), 2621-2624, https://doi.org/10.1103/PhysRevB.46.2621.

33. Diarra, M. et al. (2007). Ionization energy of donor and acceptor impurities in semiconductor nanowires: Importance of dielectric confinement. Phys. Rev. B, 75(4), 045301(1)-045301(4), https://doi.org/10.1103/physrevb.75.045301.

34. Aharonyan, K. H. (2012). Screened coulomb bound states in a finite confining potential semiconductor quantum well. J. Phys. Conf. Series, 350, 012015(1)-012015(8), https://doi.org/10.1088/1742-6596/350/1/012015.

35. Jackson, J. D. (1974). Classical electrodynamics, 2nd ed. Singapore: John Wiley \& Sons. 